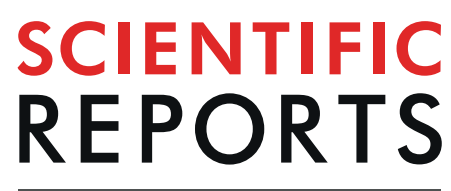

natureresearch

\title{
Spermidine protects from age- related synaptic alterations at hippocampal mossy fiber-CA3 synapses
}

\author{
Marta Maglione $e^{1,2,3}$, Gaga Kochlamazashvili2 ${ }^{2}$, Tobias Eisenberg $\mathbb{1}^{4,5}$, Bence Rácz $\mathbb{1}^{6}$, \\ Eva Michael ${ }^{1,3}$, David Toppe ${ }^{1,3}$, Alexander Stumpf ${ }^{3,7}$, Alexander Wirth ${ }^{8}$, André Zeug ${ }^{8}{ }^{8}$, \\ Franziska E. Müller ${ }^{8}{ }^{8}$, Laura Moreno-Velasquez ${ }^{3,7}$, Rosanna P. Sammons $s^{3,7}$, \\ Sebastian J. Hofer $\mathbb{1}^{4,5}$, Frank Madeo ${ }^{4,5}$, Tanja Maritzen ${ }^{2}$, Nikolaus Maier ${ }^{3,7}$, \\ Evgeni Ponimaskin $\mathbb{1}^{8}{ }^{8}$, Dietmar Schmitz ${ }^{3,7}$, Volker Haucke $\mathbb{1}^{1,2,3^{*}}$ \& Stephan J. Sigrist ${ }^{1,3^{*}}$
}

Aging is associated with functional alterations of synapses thought to contribute to age-dependent memory impairment (AMI). While therapeutic avenues to protect from AMI are largely elusive, supplementation of spermidine, a polyamine normally declining with age, has been shown to restore defective proteostasis and to protect from AMI in Drosophila. Here we demonstrate that dietary spermidine protects from age-related synaptic alterations at hippocampal mossy fiber (MF)-CA3 synapses and prevents the aging-induced loss of neuronal mitochondria. Dietary spermidine rescued age-dependent decreases in synaptic vesicle density and largely restored defective presynaptic MF-CA3 long-term potentiation (LTP) at MF-CA3 synapses (MF-CA3) in aged animals. In contrast, spermidine failed to protect CA3-CA1 hippocampal synapses characterized by postsynaptic LTP from age-related changes in function and morphology. Our data demonstrate that dietary spermidine attenuates ageassociated deterioration of MF-CA3 synaptic transmission and plasticity. These findings provide a physiological and molecular basis for the future therapeutic usage of spermidine.

Aging is the major risk factor driving age-dependent memory impairment (AMI) even in the absence of neurodegenerative diseases, representing one of the top health burden of the $21^{\text {st }}$ century ${ }^{1}$. Rather than gross neuronal loss characteristic of neurodegenerative diseases ${ }^{2}$, AMI is associated with subtle but selective and region-specific changes such as dysregulation in synapse number and structure, dendrite morphology, cellular connectivity and/ or $\mathrm{Ca}^{2+}$ homeostasis ${ }^{3-5}$. Aging has also been postulated to be associated with a decline in autophagy/lysosomal proteolysis, a cytoprotective degradation pathway responsible for the turnover of long-lived aggregate-prone proteins and damaged organelles (importantly mitochondria). Autophagy is considered to exert protective functions against aging and age-associated diseases ${ }^{6}$. Irrespective of the exact and potentially complex relation of aging and protective programs such as autophagy, stimulation of autophagy has been proposed to protect from aging effects in various models ${ }^{7-9}$. We have previously demonstrated that feeding fruit flies with the naturally occurring polyamine spermidine, whose levels decline with age in a broad spectrum of organisms likely including humans $s^{7,9-12}$, protects from AMI. Spermidine supplementation counteracts ultrastructural and functional changes at aging synapses via a mechanism that requires a functional autophagy machinery ${ }^{9,11}$. First analysis in aging mice has shown that spermidine feeding prolongs life span and exerts cardioprotective effects ${ }^{7,13}$ by

\footnotetext{
${ }^{1}$ Department of Biology, Chemistry, Pharmacy, Freie Universität Berlin, 14195, Berlin, Germany. ${ }^{2}$ Department of Molecular Pharmacology and Cell Biology, Leibniz Forschungsinstitut für Molekulare Pharmakologie (FMP), 13125 , Berlin, Germany. ${ }^{3}$ NeuroCure Cluster of Excellence, Charité Universitätsmedizin, 10117, Berlin, Germany. ${ }^{4}$ Institute of Molecular Biosciences, NAWI Graz, University of Graz, 8010, Graz, Austria. ${ }^{5}$ BioTechMed-Graz, 8010, Graz, Austria. ${ }^{6}$ Department of Anatomy and Histology, University of Veterinary Medicine Budapest, 1078, Budapest, Hungary. ${ }^{7}$ Charité - Universitätsmedizin Berlin, corporate member of Freie Universität Berlin, Humboldt-Universität zu Berlin, and Berlin Institute of Health, 10117, Berlin, Germany. ${ }^{8}$ Cellular Neurophysiology, Hannover Medical School, 30625, Hannover, Germany. *email: Haucke@fmp-berlin.de; stephan.sigrist@fu-berlin.de
} 
$\mathbf{a}$

Young (5 MO)

Aged Spd- (24 MO) Aged Spd+ (18+6 MO)
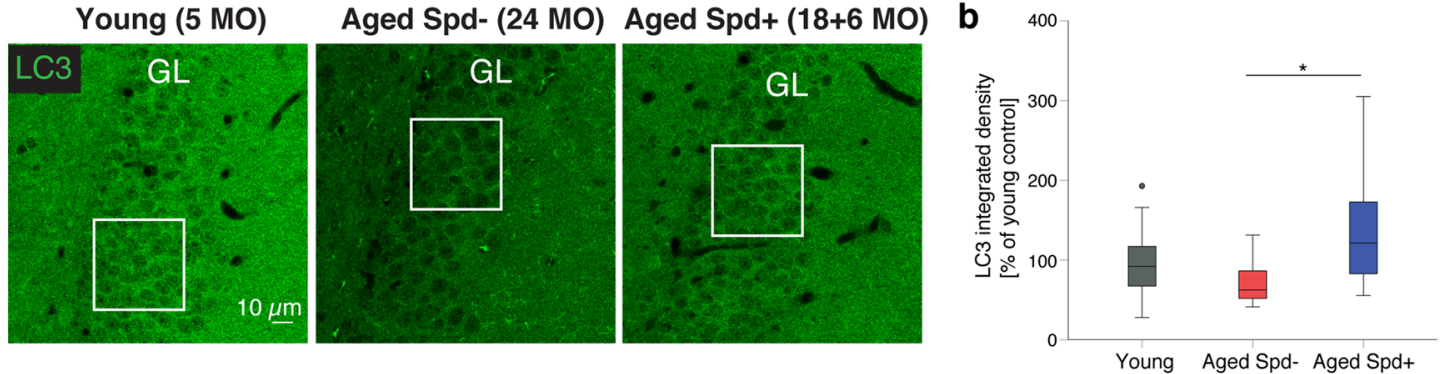

C
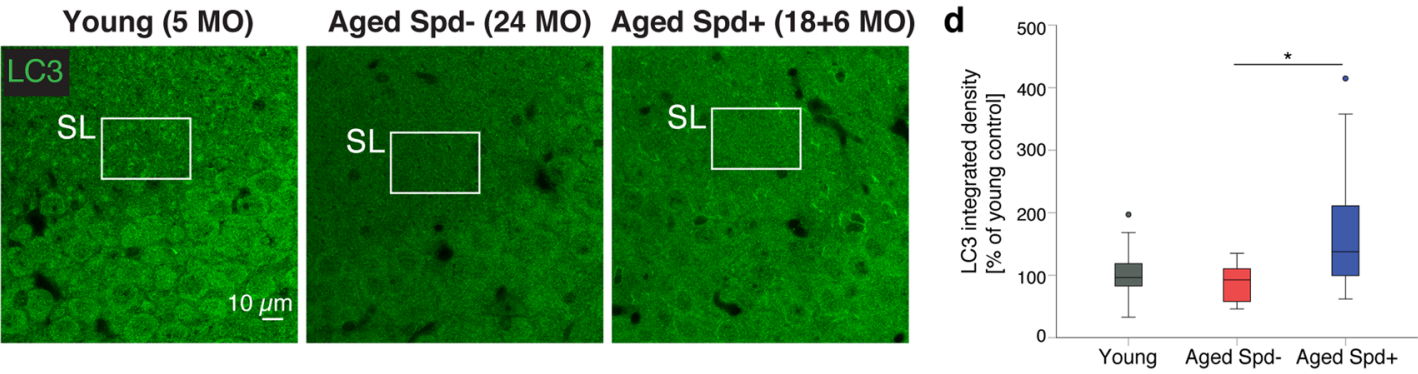

e
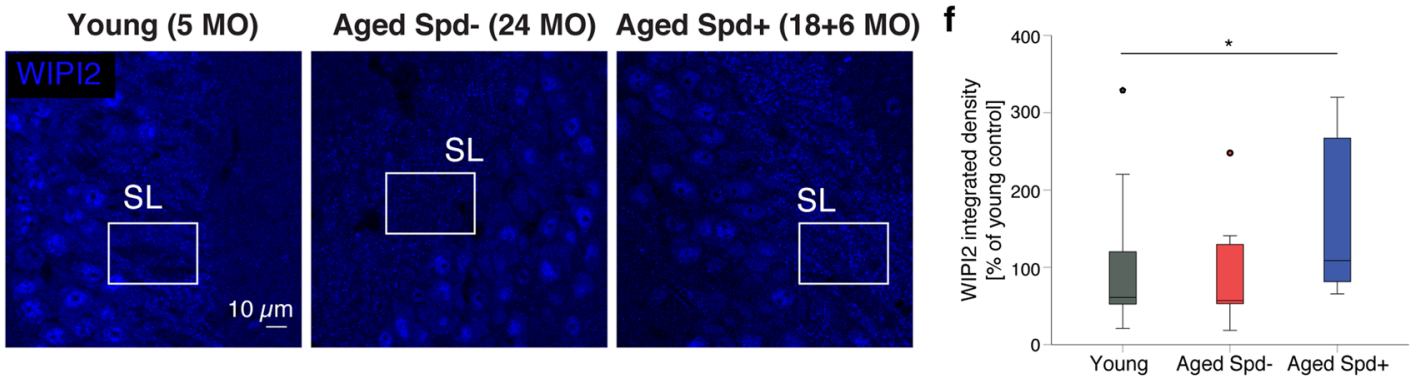

g

Young (5 MO)

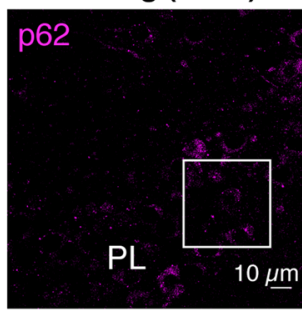

Aged Spd- (24 MO) Aged Spd+ (18+6 MO)
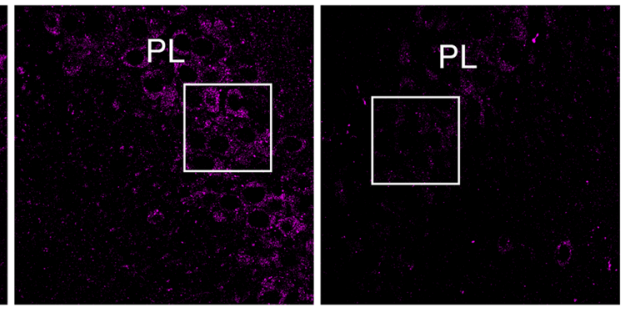

h
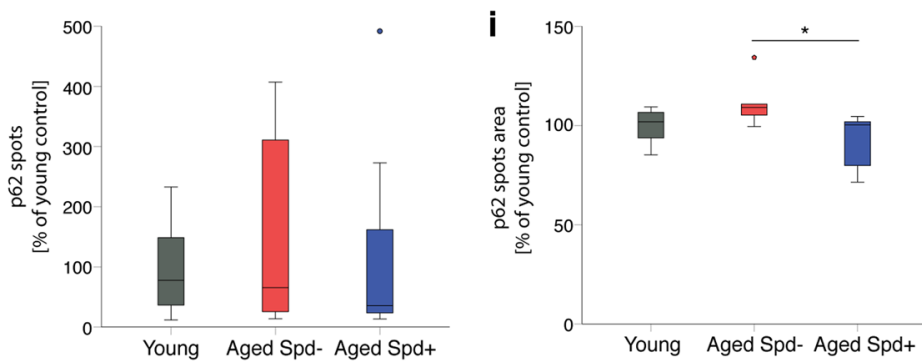

Figure 1. Spermidine treatment increases LC3 and WIPI2 levels while reducing p62 in aged hippocampal neurons. (a) Confocal images of LC3 immunoreactivity in the dentate gyrus granule cell layer (GL) of young (5 months old), aged control ( 24 months old) and aged spermidine treated (18+6 months) mice. (b) GL LC3 integrated density (young: $100 \pm 13, \mathrm{n}=14$ mice; aged Spd-: $72 \pm 9, \mathrm{n}=10$ mice; aged Spd $+: 134 \pm 21$, $\mathrm{n}=12$ mice, aged Spd- versus aged Spd $+: \mathrm{p}=0.026907$, one-way ANOVA with Tukey post-hoc test). (c) Representative confocal images of LC3 immunoreactivity in the CA3 stratum lucidum (SL) of young, aged control and aged spermidine treated mice. (d) SL LC3 integrated density (young: $100 \pm 12, \mathrm{n}=14$ mice; aged Spd-: $88 \pm 9, \mathrm{n}=10$ mice; aged Spd $+: 172 \pm 32, \mathrm{n}=12$ mice; aged Spd- versus aged Spd +: $\mathrm{p}=0.013770$, Kruskal-Wallis test followed by Mann Whitney U test with Bonferroni correction with $\alpha$ set to 0.016667). Results represent 1-2 technical replicates. (e) Confocal images of WIPI2 immunoreactivity in the CA3 SL of young, aged control and aged spermidine treated mice. (f) SL WIPI2 integrated density (young: $100 \pm 24, \mathrm{n}=14$ mice; aged Spd-: $88 \pm 22, \mathrm{n}=10$; aged $\mathrm{Spd}+: 159 \pm 29, \mathrm{n}=12$ mice; young versus aged $\mathrm{Spd}+: \mathrm{p}=0.012670$, 
Kruskal-Wallis test followed by Mann Whitney U test with Bonferroni correction with $\alpha$ set to 0.016667). (g) Confocal images of p62 immunoreactivity in the CA3 pyramidal layer (PL) of young, aged control and aged spermidine treated mice. (h) PL p62 spots number normalized to young controls (young: $100 \pm 24, \mathrm{n}=10$ mice; aged Spd-: $148 \pm 68, \mathrm{n}=6$ mice; aged $\mathrm{Spd}+: 130 \pm 69, \mathrm{n}=7$ mice). (i) PL p62 spots area normalized to young controls (young: $100 \pm 3$; aged Spd-: $111 \pm 5$; aged $S p d+: 91 \pm 5$; aged Spd- versus aged $\mathrm{Spd}+: \mathrm{p}=0.013986$, Kruskal-Wallis test followed by Mann Whitney U test with Bonferroni correction with $\alpha$ set to 0.016667). ROIs indicate the area quantified within each image. ${ }^{*} \mathrm{p}<0.05$. Values represent mean \pm SEM. Graphs show medians, interquartile ranges and $\mathrm{min} / \mathrm{max}$ values. Circles are outliers and pentagons are extremes.

autophagic reprogramming ${ }^{13,14}$. Interestingly, a recent pilot trial study indicates that spermidine might exert beneficial effects on cognitive performance in elderly humans at risk of dementia ${ }^{15}$. Thus, taken together, spermidine is a promising candidate for anti-AMI interventions.

Synaptic plasticity in the hippocampus, a pivotal cortical area for learning and memory, is particularly sensitive to aging ${ }^{16,17}$ and age-induced impairment of hippocampus-dependent memories in mice has been suggested to be linked to the downregulation of select autophagy factors ${ }^{8,18}$. Two well-studied types of hippocampal synapses are the (i) mossy fiber (MF)-CA3 synapses formed by dentate granule cell (DG) axons onto CA3 pyramidal neurons and (ii) the CA3-CA1 synapses of the Schaffer collateral pathway. MF-CA3 and CA3-CA1 Schaffer collateral synapses display distinct pre- and postsynaptic forms of long-term potentiation (LTP), respectively ${ }^{19}$, and, hence, might be differentially affected by dietary spermidine in aging animals.

Here we show that both types of hippocampal synapses suffer from an aging-induced decrease in synaptic vesicle (SV) density and mitochondria abundance. Dietary spermidine supplementation protected from age-related synaptic alterations and partially restored defective LTP selectively at hippocampal mossy fiber (MF)-CA3 but not CA3-CA1 synapses while preventing the aging-induced loss of neuronal mitochondria at both types of synapses. Our results unravel synapse-specific beneficial effects of spermidine at hippocampal synapses characterized by differential synaptic plasticity, thereby providing information for the future development of potential therapies against AMI.

\section{Results}

Dietary spermidine supplementation age-protects autophagy in the mouse hippocampal CA3 area. Based on previous studies demonstrating that spermidine supplementation stimulates autophagy $y^{7,20}$, we set up several independent cohorts of aged mice undergoing a six-months long spermidine treatment in their drinking water, starting at 18 months of age. To determine if spermidine (Spd) treatment was able to protect from aging-induced decline of autophagy, we analyzed the expression levels of the key autophagy protein LC3 in the hippocampus of young, aged Spd $-(24$ months old $)$ and aged Spd $+(18+6$ months $)$ mice. We focused on hippocampal granule cells that form MF synapses onto CA3 pyramidal neurons. In the dentate gyrus granule cell body layer, aging tended to decrease LC3 levels. Importantly, these levels were significantly increased by spermidine treatment (Fig. 1a,b). Similar observations were made in the CA3 stratum lucidum area, where granule cells form MF-CA3 synapses. Akin to the dentate gyrus LC3 levels also tended to decline with age in the synaptic CA3 area, while spermidine treatment led to a significant increase in LC3 expression (Fig. 1c,d). These data suggest that dietary spermidine indeed boosts the steady-state levels of the key autophagy protein LC3 in hippocampal granule cells.

Next, we analyzed the expression levels of WIPI2, an essential autophagy protein whose overexpression restores autophagosome biogenesis in aged dorsal root ganglion neurons ${ }^{21}$. Notably, WIPI2 immunoreactivity appeared particularly pronounced in the stratum lucidum (SL), e.g. a region where MFs are located (Fig. 1e). In the CA3 SL spermidine treatment tended to increase WIPI2 levels compared to identically aged controls (Fig. 1e,f) and WIPI2 levels were significantly upregulated in the spermidine treated cohort in comparison to young control mice (Fig. 1e,f).

Reduced autophagic protein turnover is typically associated with the accumulation of p62, an autophagy receptor for aggregated proteins that itself is a substrate for autophagy ${ }^{8}$. Although the number and size of p62 puncta was highly variable in aged mice, we found that spermidine treatment significantly reduced the p62 spot area as compared to aged matched non-spermidine treated controls (Fig. 1g-i). Collectively, these data suggest that dietary spermidine may indeed promote autophagy in the mouse hippocampal CA3 area in vivo.

Dietary spermidine stabilizes and protects mitochondria at aging hippocampal synapses. Mitophagy, a specialized form of selective autophagy, is an essential mechanism for the long-term maintenance and protection of mitochondrial abundance and functional integrity. Spermidine previously was found to activate the degradation of dysfunctional mitochondria by mitophagy in the heart ${ }^{7}$. We therefore ultrastructurally analyzed mitochondria in presynaptic terminals of MF-CA3 synapses (Fig. 2a-d). Although aging did not alter the percentage of mossy fiber boutons containing at least one mitochondrion (Fig. S1a), the density of mitochondria in aged mossy fiber boutons was significantly decreased compared to MFBs of young adult mice (Fig. 2b), confirming previous observations at mossy fiber boutons of aged rats ${ }^{22}$. Dietary spermidine rescued the age-dependent decrease in mitochondria density at mossy fiber boutons (Fig. 2b), without affecting the fraction of boutons containing mitochondria (Fig. S1a). Interestingly, adult MF terminals often displayed several mitochondria arranged in local networks (Fig. S1b), while in aged mossy fiber boutons such networks tended to decrease (Fig. S1b). Instead, individual mitochondria appeared enlarged, a feature typically associated with a dysfunctional state (Fig. 2a-d). Aging previously was reported to lead to increases in mitochondrial size ("swelling") ${ }^{23}$, a feature that we also observed in our aged cohort (Fig. 2a,d). Spermidine treatment appeared to 
a

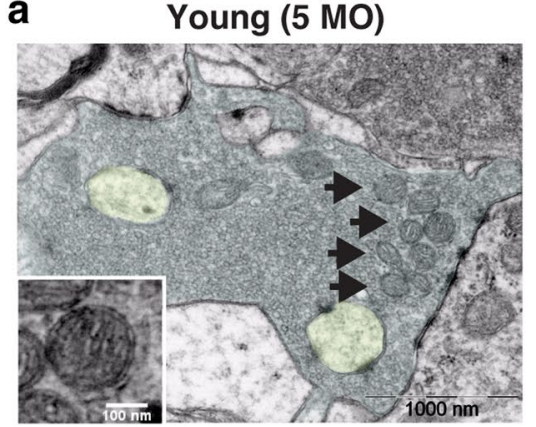

b

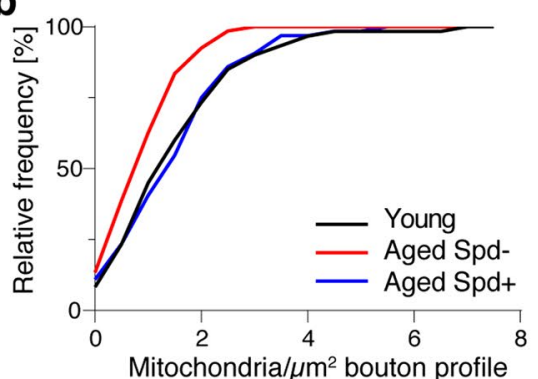

e

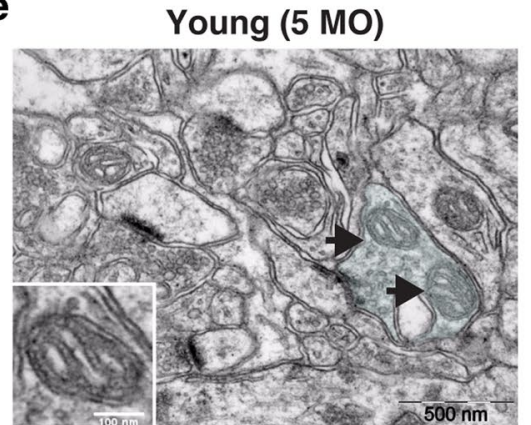

$\mathbf{f}$

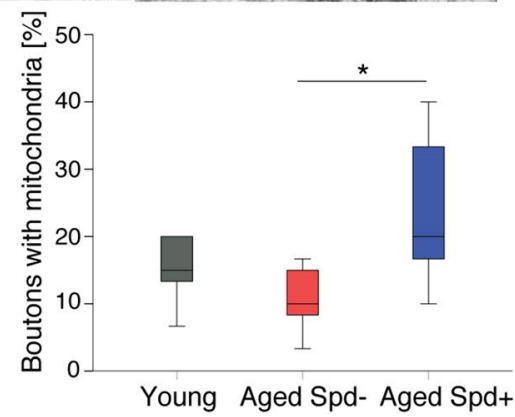

Aged Spd- (24 MO)

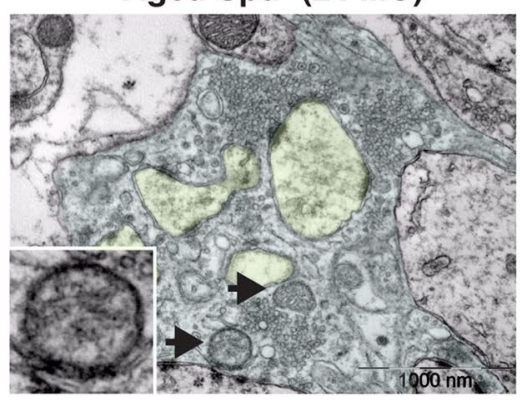

C $30-$

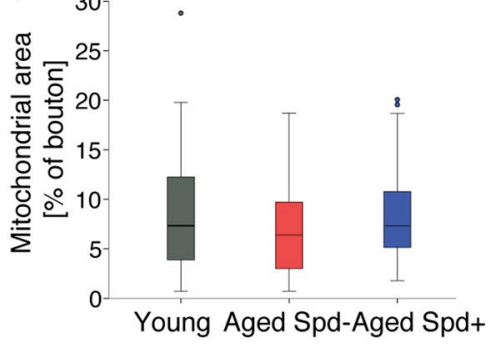

Aged Spd- (24 MO)
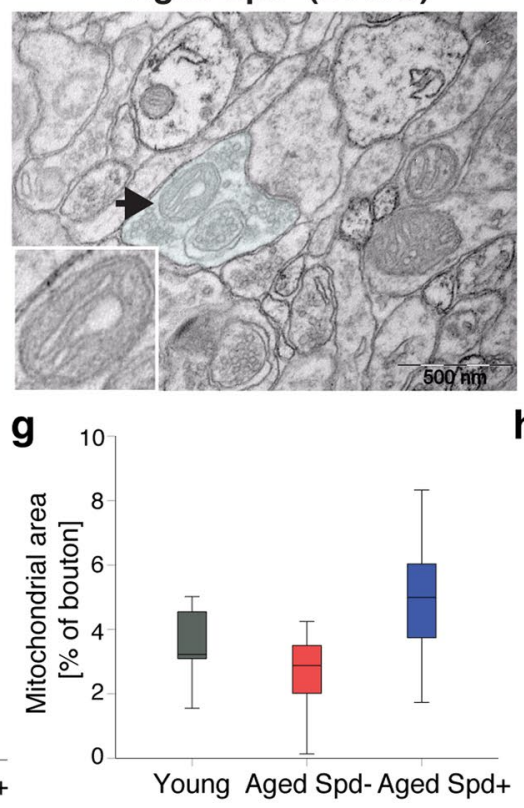

Aged Spd+ (18+6 MO)
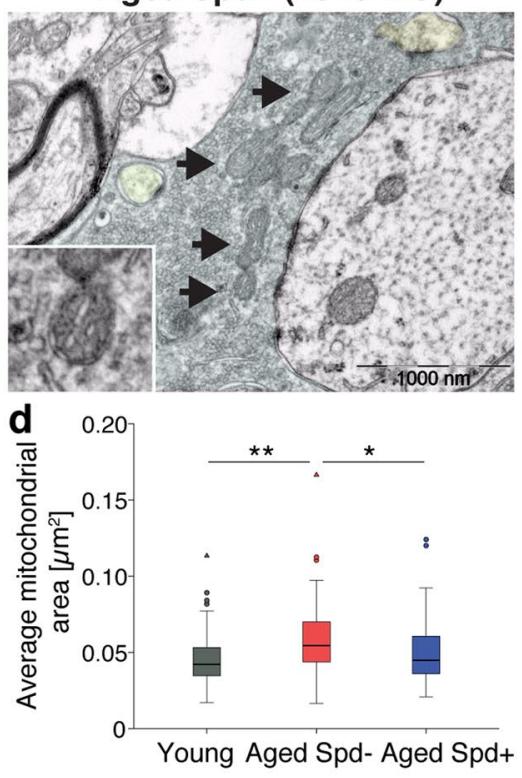

Aged Spd+ (18+6 MO)
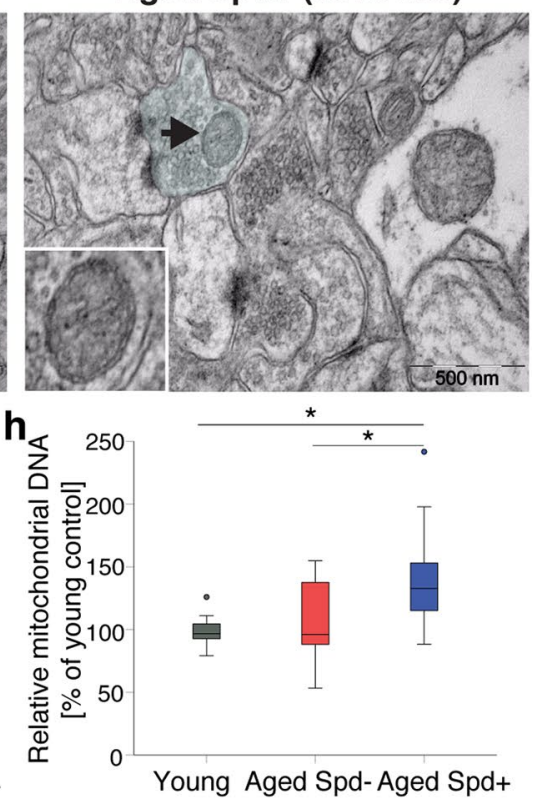

Figure 2. Spermidine protects from age-induced alterations in mitochondria at MF-CA3 synapses by increasing mitochondrial mass. (a-d) Ultrastructural analysis of Mossy Fiber (MF) terminals. MF bouton area is highlighted in light blue, spiny excrescences in yellow. (b) Mitochondria density in MF boutons $(\mathrm{p}=0.0046$, aged Spd-:1.04 $\pm 0.09, \mathrm{n}=67$ boutons, aged $\mathrm{Spd}+: 1.65 \pm 0.14, \mathrm{n}=64$ boutons; $\mathrm{p}=0.0142$, young: $1.69 \pm 0.16, \mathrm{n}=60$ boutons, versus aged $\mathrm{Spd}-, 6 \mathrm{mice}$ /group, two samples Kolmogorov-Smirnov test with Bonferroni correction with $\alpha$ set to 0.016667 ). (c) MF bouton area covered by mitochondria (young: $8.5 \pm 0.8, \mathrm{n}=55$ boutons; aged $\mathrm{Spd}-: 6.9 \pm 0.6, \mathrm{n}=58$ boutons; aged $\mathrm{Spd}+: 8.4 \pm 0.6, \mathrm{n}=56$ boutons, 6 mice/ group). (d) Average visible mitochondrial area ( $\mathrm{p}=0.011849$, aged $\mathrm{Spd}-: 0.059 \pm 0.003, \mathrm{n}=58$ boutons, aged Spd $+: 0.050 \pm 0.003, n=56$ boutons; $\mathrm{p}=0.000580$, young: $0.046 \pm 0.002, \mathrm{n}=55$ boutons, versus aged Spd-, 6 mice/group, Kruskal-Wallis test followed by Mann Whitney U test with Bonferroni correction with $\alpha$ set to 0.016667$)$. (e-g) Ultrastructural analysis of presynaptic CA3-CA1 terminals. (f) Percentage of CA3-CA1 presynaptic terminals containing at least one mitochondrion (young: $15 \pm 2, \mathrm{n}=6$ mice, 180 boutons; aged Spd-: $11 \pm 2, \mathrm{n}=7$ mice, 210 boutons; aged Spd $+: 23 \pm 5 \mathrm{n}=6$ mice, 180 boutons; aged Spd - versus aged Spd $+: \mathrm{p}=0.023280$, one-way ANOVA with Tukey post-hoc test). (g) Percentage of bouton area covered by mitochondria (young: $3.4 \pm 0.5, \mathrm{n}=6$ mice, 180 boutons analyzed; aged $\mathrm{Spd}-: 2.6 \pm 0.5, \mathrm{n}=7$ mice, 210 boutons; aged Spd $+: 4.9 \pm 0.9, \mathrm{n}=6$ mice, 180 boutons). (h) Relative mitochondrial DNA content in brains of young $(100 \pm 4, \mathrm{n}=9$ mice $)$, aged Spd $-(106 \pm 9, \mathrm{n}=12$ mice $)$ and aged Spd + mice $(144 \pm 14, \mathrm{n}=10$ mice), normalized to young controls (young versus aged $\mathrm{Spd}+: \mathrm{p}=0.021083$; aged $\mathrm{Spd}+$ versus aged Spd-: 
$\mathrm{p}=0.036320$, one-way ANOVA with Tukey post-hoc test). Relative mitochondrial DNA abundance was determined by measuring mitochondrial cytochrome B DNA content relative to nuclear DNA content ( $\beta$ actin). $* \mathrm{p}<0.05, * * \mathrm{p}<0.01$. Values represent mean \pm SEM. Graphs show medians, interquartile ranges and $\mathrm{min} / \mathrm{max}$ values. Circles are outliers and triangles are extremes. Black arrows indicate mitochondria.

protect from this age-induced swelling phenotype and tended to rescue network formation (Figs. 2a,d and S1b). When analyzing the relative bouton area covered by mitochondria in presynaptic MF terminals, we observed a trend towards rejuvenated values in the spermidine supplemented animals (Fig. 2c), though not to statistical significance. To see whether a similar protective effect of spermidine can be observed at other types of hippocampal synapses, we analyzed mitochondrial abundance at CA3-CA1 presynaptic terminals (Fig. 2e-g). At this synapse type, age tended to decrease the number of boutons containing at least one mitochondrion (Fig. 2f). Spermidine treatment significantly increased the fraction of mitochondria-containing boutons (Fig. 2f) and also tended to increase the bouton area covered by mitochondria (Fig. $2 \mathrm{~g}$ ).

Finally, we asked whether the observed increase in presynaptic mitochondrial abundance by spermidine treatment was associated with an overall increase in mitochondrial mass. To probe this, we performed quantitative real time qPCR to measure mitochondrial DNA content of young, aged mock, and aged spermidine-treated brain tissue (Fig. 2h). Analysis of the abundance of mitochondrial DNA (normalized to nuclear $\beta$ actin DNA) revealed a high variability in aged mice in comparison to young controls. Spermidine treatment significantly increased the relative mitochondrial DNA abundance in comparison to aged mice and young controls (Fig. $2 \mathrm{~h}$ ), indicating that spermidine supplementation can indeed increase net mitochondrial mass.

Taken together, our analyses demonstrate that chronic dietary spermidine supplementation results in age-protection of mitochondria at sites of neurotransmitter release of both MF-CA3 and CA3-CA1 synapses via an overall increase in mitochondrial mass.

Aging effects on MF-CA3 synapse ultrastructure are responsive to spermidine supplementation. Next we asked whether aging promotes changes in ultrastructure at mossy fiber synapses onto CA3 pyramidal neurons (MF-CA3; Fig. 3) and CA3-CA1 synapses (Fig. 4). MF-CA3 synapses display multiple release sites, large paired-pulse and frequency facilitation ${ }^{19,24,25}$ and marked synaptic plasticity of presynaptic origin ${ }^{19,26}$. MF-CA3 synapses thus markedly differ from CA3-CA1 synapses, the vast majority of which form a single synaptic contact ${ }^{27}$. Structural changes at MF-CA3 release sites during aging might therefore conceivably underlie age-dependent alterations in synaptic plasticity at this synapse. EM analysis of the ultrastructure of aged hippocampal MF-CA3 synapses revealed a significant reduction in synaptic vesicle (SV) density by approximately $30 \%$ when compared to synapses from young control mice, while the area occupied by mossy fiber boutons remained unchanged (Fig. $3 \mathrm{a}-\mathrm{c}$ ), similar to previous reports for mossy fiber boutons in aged rats ${ }^{22}$. While aging did not significantly alter the density of active zones (Fig. 3d), the length of individual active zones also significantly decreased with age (Fig. S2). Importantly, spermidine treatment for 6 months rescued the age-dependent reduction in SV density of aged MF-CA3 synapses to the density observed in young animals (Fig. 3b). Spermidine did not affect active zone number or active zone length when compared to age-matched controls (Figs. 3d, S2).

To see whether a similar protective effect of spermidine is observed at other types of hippocampal synapses, we analyzed the ultrastructure of CA3-CA1 presynaptic terminals (Fig. 4a-d). This widely investigated synapse executes long-term synaptic plasticity postsynaptically ${ }^{28}$. Consistent with our findings at MF-CA3 synapses, we found that aging decreased SV density at CA3-CA1 synapses (Fig. 4b). In contrast to MF-CA3 synapses treatment with spermidine for 6 months did not rescue these changes (Fig. 4a,b). The visible presynaptic bouton profile tended to increase in aged control and spermidine treatment significantly increased it (Fig. 4c), suggesting that spermidine may not be able to counteract aging-induced alterations in presynaptic bouton size of CA3-CA1 synapses. Similarly, aging tended to be associated with a slightly increased active zone (AZ) length, which could not be reversed by spermidine treatment (Fig. $4 \mathrm{~d}$ ).

Collectively, these data show that dietary spermidine supplementation selectively prevents aging-induced loss of SVs at MF-CA3 but not CA3-CA1 synapses in the mouse hippocampus.

Dietary spermidine age-protects long-term plasticity specifically at MF-CA3 synapses. Given these encouraging results, we investigated whether the observed protective effects of spermidine with respect to MF-CA3 synapse ultrastructure would translate into the preservation of synaptic function and plasticity. We therefore analyzed long-term potentiation (LTP), a cellular paradigm for information storage, learning and memory $^{28}$, at MF-CA3 synapses. LTP has been reported to decline with age at hippocampal synapses ${ }^{29,30}$, including MF-CA3 synapses ${ }^{31}$. Indeed, when we analyzed acute hippocampal slices from aged mice (24 months) by field recordings (Fig. 5a), we observed a severe reduction of MF-LTP in comparison to young adult mice (5 months; Fig. 5b,c). Frequency facilitation, a form of short term plasticity pronounced at MF-CA3 synapses in which a switch from low to modest stimulation frequencies lead to an increase in synaptic strength ${ }^{19}$, was unaltered (Fig. S3). Importantly, we found that spermidine supplementation significantly rescued the age-dependent decrease of MF-LTP in 24 months-old animals (Fig. 5b,c). When we analyzed EPSP/fiber volley curves, we observed an age-dependent increase in basal neurotransmission at MF-CA3 synapses that, similar to LTP, was rescued to juvenile levels by spermidine treatment (Fig. 5d,e). As a control we also studied CA3-CA1 synapses that exhibit largely postsynaptic forms of LTP. Consistent with previous studies ${ }^{29,30}$, we observed a trend towards lower LTP of fEPSP slopes at CA3-CA1 synapses of aged animals (Fig. 6a,b). Chronic spermidine administration did not rescue this trend towards an age-dependent decrease in CA3-CA1 LTP (Fig. 6b), consistent with the lack of effect of spermidine with respect to SV density (Fig. 4b). When we analyzed CA3-CA1 EPSP/fiber volley 
a

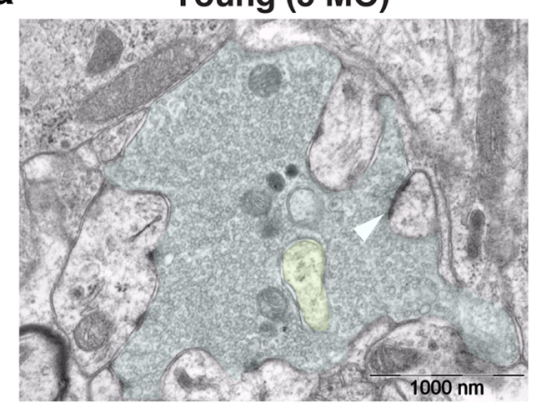

b

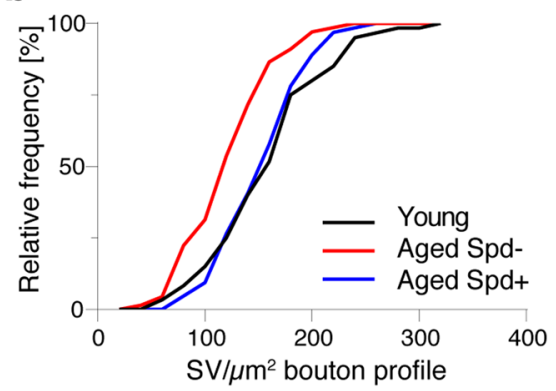

Aged Spd- (24 MO)

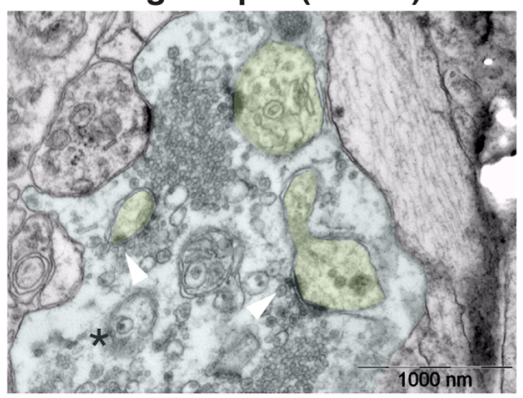

C

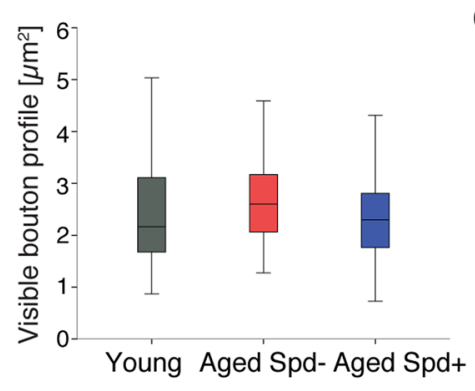

Aged Spd + (18+6 MO)

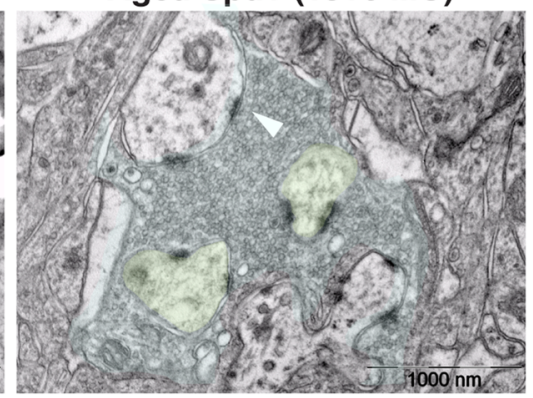

d

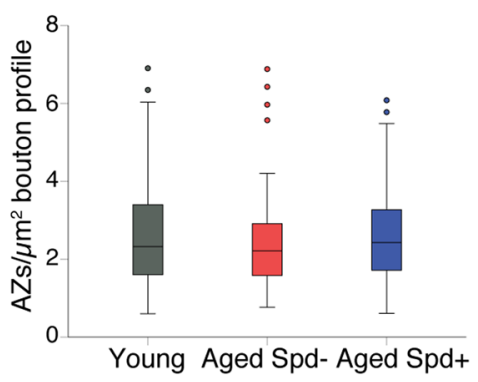

Figure 3. Spermidine treatment rescues the age-dependent decrease in SV density selectively at MFCA3 synapses. (a-d) Ultrastructural analysis of Mossy Fiber (MF) terminals. (a) Representative electron micrographs of MF boutons. Note the reduction in SV density in an aged Spd- bouton (black asterisk). MF bouton visible area is highlighted in light blue, spiny excrescences are highlighted in yellow. (b) Synaptic vesicles (SV) density ( $\mathrm{p}=0.0004$, aged $\mathrm{Spd}-: 128.26 \pm 5.11, \mathrm{n}=67$ boutons, versus aged $\mathrm{Spd}+: 159.43 \pm 5.05, \mathrm{n}=64$; $\mathrm{p}=0.0008$, young: $163.03 \pm 6.86, \mathrm{n}=60$, versus aged $\mathrm{Spd}-; 6$ mice/group; two samples Kolmogorov-Smirnov test Bonferroni corrected with $\alpha$ set to 0.016667$)$. (c) Visible bouton profile indicating the synaptic bouton area visible within each image (aged Spd-: $2.69 \pm 0.09$, aged Spd $+: 2.34 \pm 0.10$, young: $2.43 \pm 0.13$ ). (d) Active zone (AZ) density (aged Spd-: $2.41 \pm 0.15$, aged Spd $+: 2.62 \pm 0.16$, young: $2.65 \pm 0.19$ ). Arrowheads indicate active zones, note an active zone with drastically reduced neighboring SVs in the representative electron micrograph of an aged control mouse. Values represent mean \pm SEM. Graphs show medians, interquartile ranges and min/ max values, circles are outliers.

curves, we did not observe any significant changes with either aging or spermidine treatment (Fig. 6c,d). Taken together, our results reveal a synapse-specific restoration of presynaptic LTP at MF-CA3 synapses by dietary spermidine in aged mice.

\section{Discussion}

Elucidating the cellular and molecular mechanisms that underlie the aging of brain function are a major challenge of neuroscience. Finding dietary regimes to fundamentally reduce the rate at which these processes occur is equally relevant. Recent reports suggest that with aging neuronal autophagy might decline $e^{6,8}$ and, importantly, that restoring hippocampal autophagy in aged mice allows for a rescue of age-induced memory impairment ${ }^{8,32}$. In the present study, we show that dietary supplementation of spermidine, a substance usually declining with age, can restore levels of core autophagic proteins. We found that spermidine treatment for 6 months starting late in life (at 18 months), a feeding scheme previously shown to extend life-span of aged mice ${ }^{7}$, enhanced clearance of the autophagy substrate p62 and increased expression of key autophagy proteins (LC3, WIPI2). These data are consistent with previous studies in other organ systems demonstrating that spermidine administration can stimulate autophagic flux $x^{20,33-35}$ and a prominent autophagic role for WIPI2 in aged neurons ${ }^{21}$. These data thus indicate that chronic spermidine supplementation might promote autophagy in the aged mouse brain.

Age-dependent cognitive decline seems to be tightly associated with subtle synaptic changes but is not mandatorily linked to neuronal cell death ${ }^{3}$, a prominent feature of neurodegenerative disorders rather than normal aging. Changes in the density of the pool of synaptic vesicles distant from active zones (e.g. the recycling and reserve pools) have been previously described at hippocampal Schaffer collateral synapses of aged rats ${ }^{36}$ and at MF-CA3 synapses ${ }^{22}$. Our data confirm that aging indeed leads to decreased SV density at both MF-CA3 and CA3-CA1 synapses (Figs. 3a,b and 4a,b). Most importantly, we found that spermidine treatment late in life could rescue the age-dependent reduction in SV density and in LTP at MF-CA3 synapses. Strikingly, this phenotype was synapse-specific as spermidine administration failed to elicit postsynaptic LTP at and to restore SV density at CA3-CA1 synapses (Figs. 5b,c and 6b). Spermidine supplementation also rescued the age-dependent increase in EPSP/fiber volley amplitudes observed at MF-CA3 synapses (Fig. 5d,e), which might indicate higher basal neurotransmission at these synapses with age, as previously reported for aged synapses in the Drosophila olfactory circuitry ${ }^{11}$. Our results are in contrast with a recent study at aged MF-CA3 synapses in rats using 4-6 week 
a

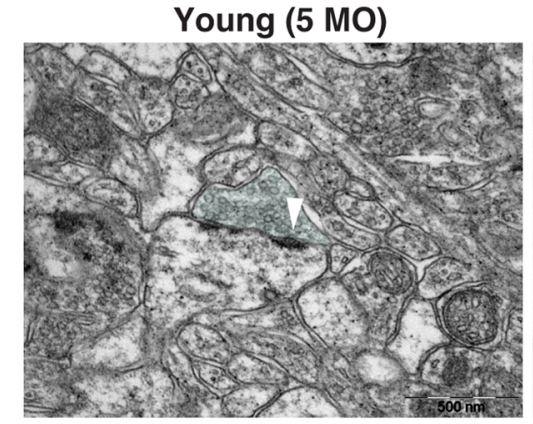

b

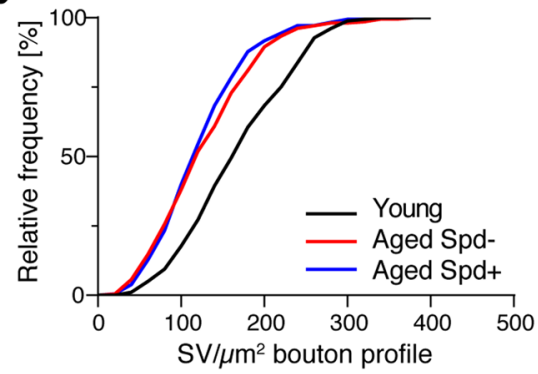

Aged Spd- (24 MO)
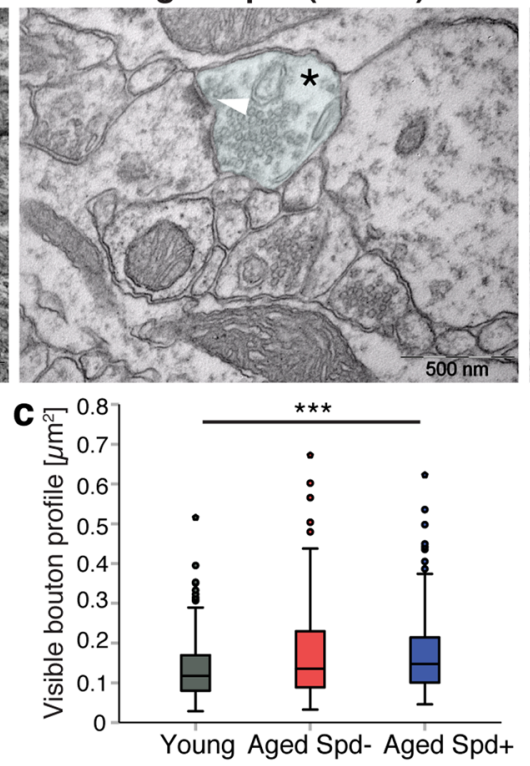

Aged Spd+ (18+6 MO)

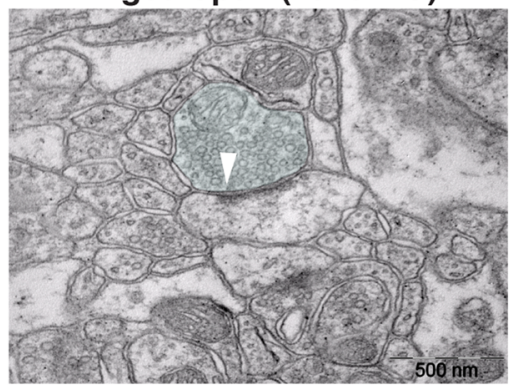

d

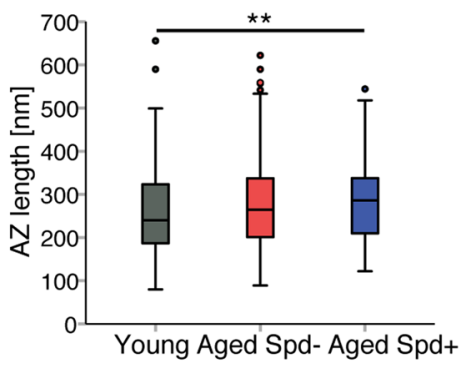

Figure 4. Spermidine treatment does not rescue the age-dependent decrease in SV density at CA3-CA1 synapses. (a-d) Ultrastructural analysis of presynaptic CA3-CA1 terminals, whose visible area is here highlighted in light blue. (a) Representative electron micrographs of CA3-CA1 synapses. Note the reduction in SV density in an aged Spd- bouton (black asterisk). (b) Synaptic vesicle (SV) density (young: 175 $\pm 5, \mathrm{n}=180$ boutons, 6 mice analyzed; aged $\mathrm{Spd}-: 136 \pm 4, \mathrm{n}=210,7$ mice; aged $\mathrm{Spd}+: 130 \pm 4, \mathrm{n}=180,6$ mice; young versus aged $\mathrm{Spd}-: \mathrm{p}<0.0001$; young versus aged $\mathrm{Spd}+: \mathrm{p}<0.0001$, two samples Kolmogorov-Smirnov test Bonferroni corrected with $\alpha$ set to 0.016667$)$. (c) Visible bouton profile indicating the synaptic bouton area visible within each image (young: $0.135 \pm 0.006$, aged Spd-: $0.166 \pm 0.008$, aged Spd +: $0.173 \pm 0.008$; young versus aged $\mathrm{Spd}+: \mathrm{p}=0.000106$, Kruskal-Wallis test followed by Mann Whitney U test Bonferroni corrected with $\alpha$ set to 0.016667 ). (d) Active zone (AZ) length (young: $260 \pm 7$, aged Spd-: $280 \pm 7$, aged Spd+: $287 \pm 7$; young versus aged $S p d+: p=0.002623$, Kruskal-Wallis test followed by Mann Whitney U test Bonferroni corrected with $\alpha$ set to 0.016667$)$. ** $\mathrm{p}<0.01, * * * \mathrm{p}<0.001$. Arrowheads indicate active zones, note an active zone with drastically reduced neighboring SVs in the representative electron micrograph of an aged control mouse. Values represent mean \pm SEM. Graphs show medians, interquartile ranges and $\mathrm{min} / \mathrm{max}$ values. Circles are outliers and pentagons are extremes.

old animals as young controls ${ }^{31}$, which might reflect developmental changes still occurring at this early age. The discrepancy might be due to the different species and age windows used.

Different from CA3-CA1 synapses, MF-CA3 synapses are characterized by presynaptic plasticity, a process controlled by presynaptic cAMP and cAMP-dependent protein kinase (PKA) via Rab3a, a SV protein that regulates SV fusion ${ }^{19,37,38}$. Interestingly, we observed similar beneficial effects of spermidine at Drosophila mushroom body synapses that are also characterized by cAMP-driven presynaptic plasticity to form new memories ${ }^{9,11,39}$. Our results might indicate that spermidine acts on a mechanism that specifically regulates the SV pool at MF terminals. This is not unlikely considering that MF-CA3 synapses are remarkably different in term of structure, release probability and plasticity from CA3-CA1 synapses ${ }^{19,24-27,40}$. MF-CA3 synapses display multiple release sites, low release probability ${ }^{41}$, strong facilitation and execute long term plasticity via presynaptic mechanisms. In contrast, CA3-CA1 synapses form a single synaptic contact and are characterized by higher release probability ${ }^{42,43}$. Past studies have demonstrated a differential regulation or role of proteins involved in SV cycling at MF-CA3 versus CA3-CA1 synapses, supporting our findings. The Rab3-interacting protein Rabphilin is a PKA effector, that controls the recovery of the ready releasable pool (RRP) of SVs following extensive synaptic activity ${ }^{44}$. Active PKA has been shown to differentially phosphorylate Rabphilin at MF $v$ s. CA3-CA1 synapses, suggesting a MF-specific mechanism regulating SVs exocytosis upon RRP depletion ${ }^{45}$. We thus speculate that spermidine might exert beneficial effects specifically at synapses executing synaptic plasticity via presynaptic mechanisms, possibly including autophagic turnover of presynaptic components. While these mechanisms may be complex, our data showing that spermidine supplementation prevents aging-induced defects in presynaptic mitochondria (Fig. 2), suggests a possible role for mitochondrial maintenance in presynaptic $\mathrm{Ca}^{2+}$ homeostasis ${ }^{46-48}$. Interestingly, spermidine has been found to increase the rate and affinity of $\mathrm{Ca}^{2+}$ uptake in brain mitochondria ${ }^{49}$. This may be of particular importance for pre- but not postsynaptic forms of LTP, i.e. at MF-CA3 synapses. Future studies will be needed to address this possibility. We would like to note that effects of spermidine on CA1 mitochondrial morphological parameters could not be observed in another cohort with very different housing conditions (smaller group size, different environmental enrichment) in a different animal facility which also showed less clear aging effects (data not shown). This suggests that environmental factors can impinge on neuronal ultrastructure of the aging brain per se or on the effects of spermidine in particular. Defining these factors should also be subject of future research. 
a

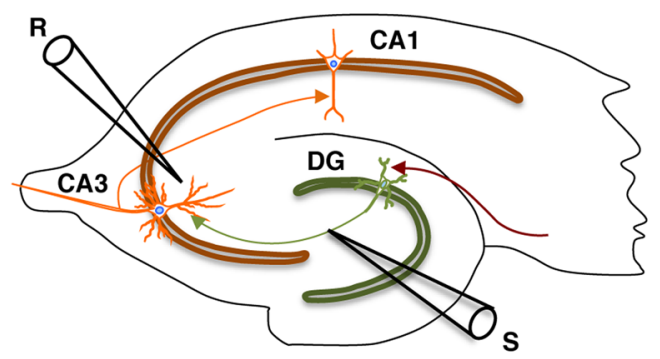

b
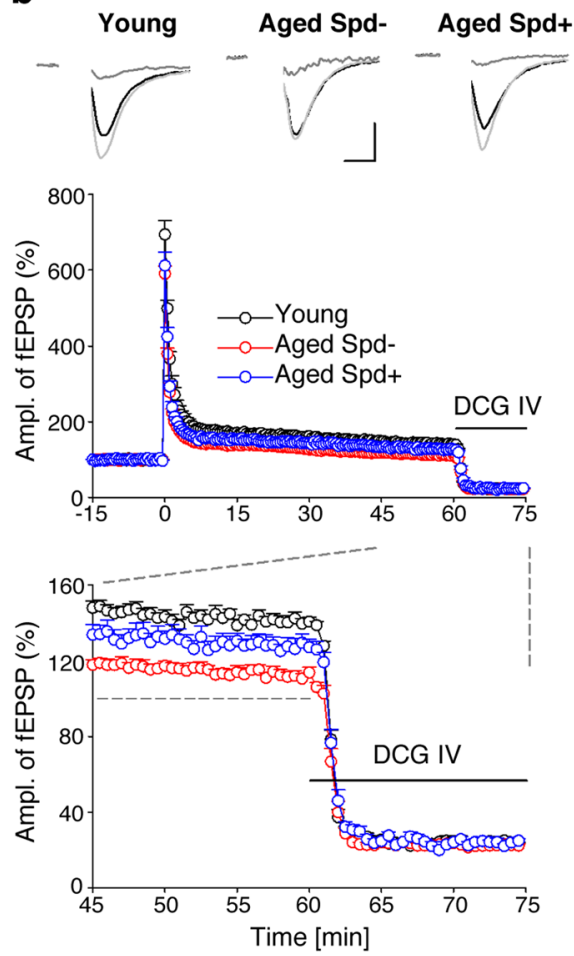

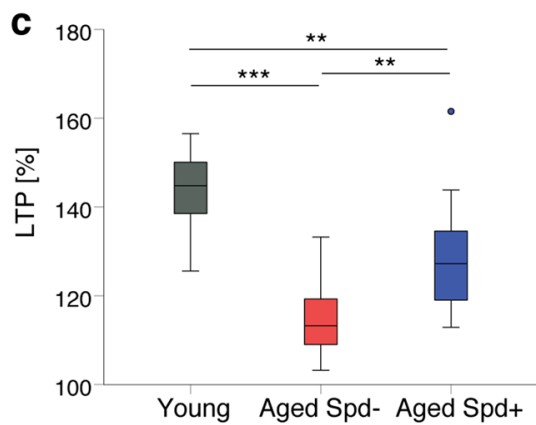

d

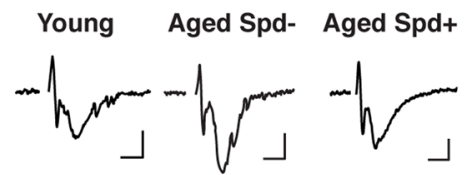

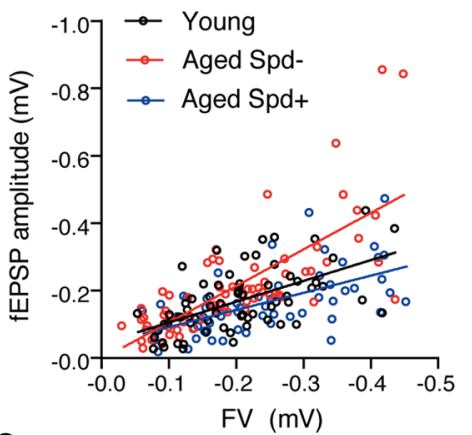

e

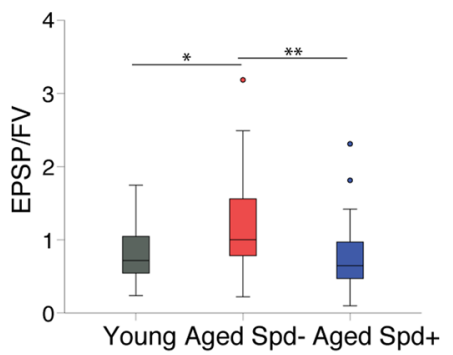

Figure 5. Spermidine treatment rescues the age-dependent decrease in LTP selectively at MF-CA3 synapses while restoring their EPSP/fiber volley curves. (a) Scheme of electrophysiological recordings at MF-CA3 synapses, depicting stimulation (S), recording (R) electrode. (b) HFS induced LTP of MF-fEPSPs in young $(143.1 \pm 2.7)$, aged $(114.9 \pm 2.5)$ and spermidine treated mice $(130.1 \pm 4.5)$ and averaged MF-fEPSPs collected before (black), 45-60 min after LTP induction (gray) and after application of the agonist of type II metabotropic glutamate receptors DCG IV $(2 \mu \mathrm{M}$; dark gray). Only responses inhibited by $70-80 \%$ and more were assumed to be elicited by mossy fiber synapses. Calibration: $0.3 \mathrm{mV}, 5 \mathrm{~ms}$. (c) LTP levels $45-60 \mathrm{~min}$ after induction. Young: $\mathrm{n}=12$ slices $\mathrm{N}=6$ mice, aged Spd-: $\mathrm{n}=13 \mathrm{~N}=7$, aged Spd $+: \mathrm{n}=10 \mathrm{~N}=6(\mathrm{p}=<0.001$, One-way RM ANOVA with Shapiro-Wilk post-hoc test: young versus aged $\mathrm{Spd}-: \mathrm{p}<0.001$, aged Spd- versus aged Spd $+: p=0.002$, young versus aged $S p d+: p=0.002$ ). (d) Correlation of MF-fEPSP amplitude and FV (fiber volley; ANCOVA: $\mathrm{p}=0.0003$ ) and representative average traces of MF-fEPSPs with FVs, scaled to similar FV amplitude. Calibration: $0.2 \mathrm{mV}, 5 \mathrm{~ms}$. Several stimulation intensities $(0-500 \mu \mathrm{A})$ were used to record different FV amplitudes (young: 67 data points; aged Spd-: 68; aged Spd+: 58). (e) Average fEPSP/FV values for each slice (young: $0.79 \pm 0.08$; aged $\mathrm{Spd}-; 1.19 \pm 0.11$; aged $\mathrm{Spd}+: 0.76 \pm 0.81$; young versus aged $\mathrm{Spd}-: \mathrm{p}=0.011342$; aged Spd - versus aged Spd $+: \mathrm{p}=0.000997$, Kruskal-Wallis test followed by Mann Whitney U test Bonferroni corrected with $\alpha$ set to 0.016667 ). Young: $\mathrm{n}=22 \mathrm{~N}=9$; aged Spd $-: \mathrm{n}=36 \mathrm{~N}=14$; aged $\mathrm{Spd}+: \mathrm{n}=33 \mathrm{~N}==16$. $* \mathrm{p}<0.05, * * \mathrm{p}<0.01, * * * \mathrm{p}<0.001$. Values and graphs as in Fig. 3 .

Taken together, we provide a direct demonstration of the beneficial effects of dietary spermidine supplementation in an electrophysiological paradigm of learning in a mammalian model. Our findings may, thus, be of importance for the development of future therapies against AMI. 
a

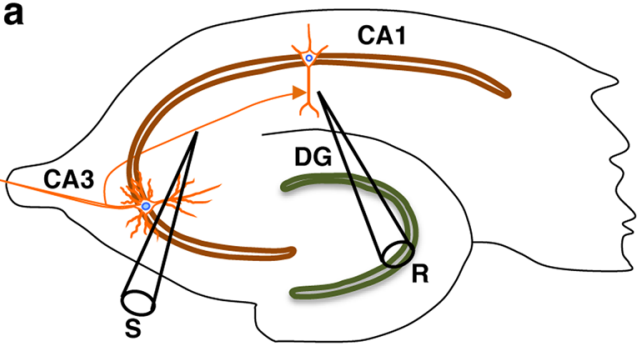

b
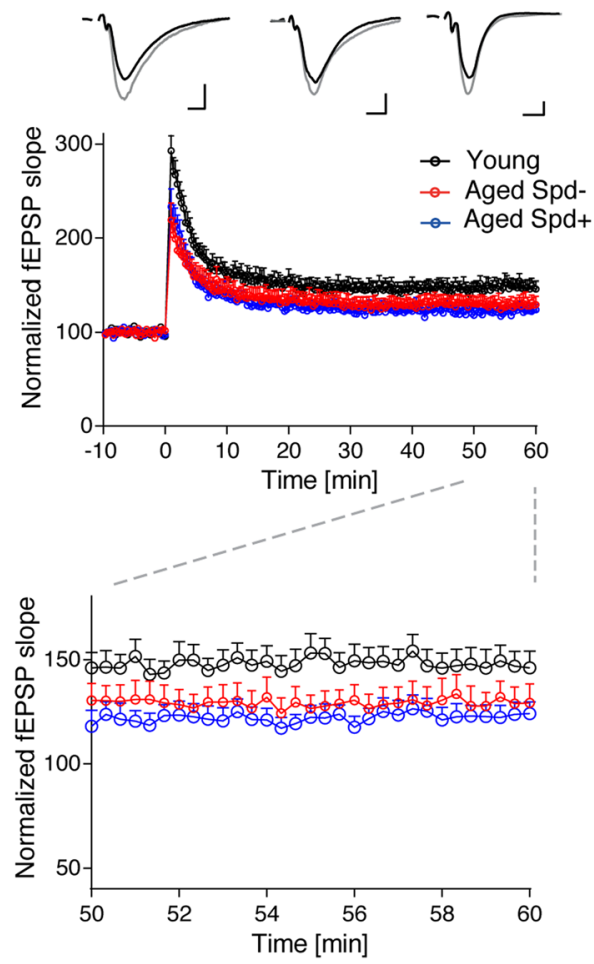

C
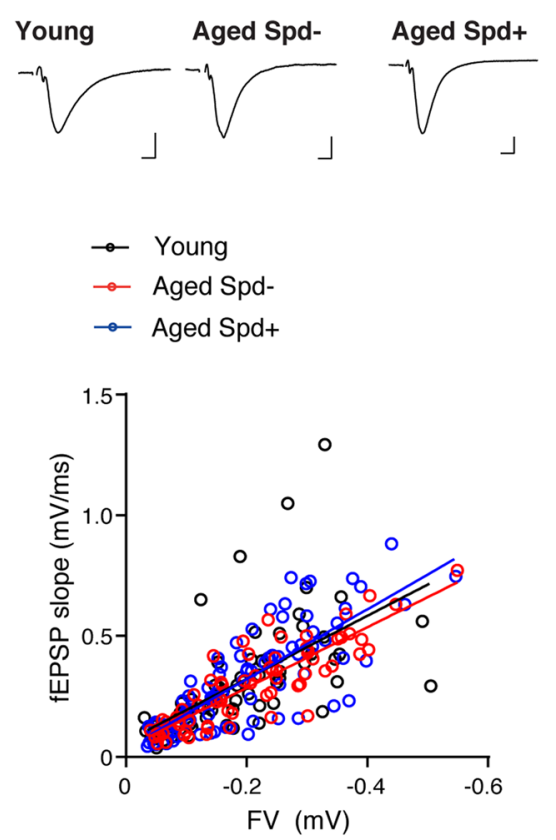

d

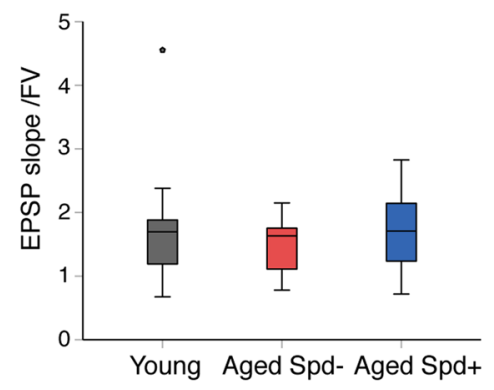

Figure 6. Spermidine treatment does not rescue the age-dependent decrease in LTP at CA3-CA1 synapses. (a) Scheme of electrophysiological recordings at hippocampal CA3-CA1 synapses, depicting stimulation (S), recording (R) electrode (Dentate gyrus - DG, CA3 and CA1). (b) Time course of LTP induction in CA3-CA1 synapses and average baseline fEPSPs (black) for $10 \mathrm{~min}$ before LTP induction and potentiated fEPSPs (gray), 50-60 min after. Young: $145.8 \pm 6.9, \mathrm{n}=13$ slices $\mathrm{N}=5$ mice; aged $\mathrm{Spd}-: 130.6 \pm 7.6, \mathrm{n}=10 \mathrm{~N}=4$; aged Spd+: $123.2 \pm 5.4, \mathrm{n}=13 \mathrm{~N}=6(\mathrm{p}=0.0504$, One-way ANOVA). Calibration: $0.2 \mathrm{mV}, 5 \mathrm{~ms}$. (c) Correlation of fEPSP slope and FV (fiber volley) at CA3-CA1 synapses (ANCOVA: $p=0.5754$ ) and average traces of CA3-CA1fEPSPs with FVs, scaled to similar FV amplitude. Calibration: $0.2 \mathrm{mV}, 5 \mathrm{~ms}$. Several stimulation intensities $(0-500 \mu \mathrm{A})$ were used to record different FV amplitudes; each data point represents the average values for one stimulation intensity (young: 72 data points; aged Spd-: 70 data points; aged Spd+: 85 data points). (d) Average CA3-CA1 fEPSP slope/FV values for each slice (young: $1.791 \pm 0.26, \mathrm{n}=13 \mathrm{~N}=5$, aged $\mathrm{Spd}-: 1.521 \pm 0.14$, $\mathrm{n}=10 \mathrm{~N}=4$, aged $\mathrm{Spd}+: 1.707 \pm 0.17, \mathrm{n}=14 \mathrm{~N}=6 ; \mathrm{p}=0.7645$, Kruskal-Wallis test). Values and graphs as in Fig. 4.

\section{Material and Methods}

Spermidine supplementation. C57BL6 WT mice were purchased from Janvier Labs (C57BL/6 J:Rj males). Spermidine supplementation at a final concentration of $3 \mathrm{mM}$ in drinking water started late in life (18 months of age) for 6 months $^{7}$. A more detail description of housing conditions is found in the Supplemental Information. Notably, the effects of spermidine on CA1 mitochondrial morphological parameters were not observed in another cohort with very different housing conditions (smaller group size, different environmental enrichment) in a different animal facility (data not shown),

All animal experiments were approved by the animal welfare committee of Charité Universitätsmedizin Berlin, Leibniz Institut für Molekulare Pharmakologie (FMP) and the Landesamt für Gesundheit und Soziales Berlin and by the Bundesministerium für Wissenschaft, Forschung und Wirtschaft, BMWFW, Austria: BMWF66.007/0011-II/3b/2013, BMWFW-66.007/0002-WF/V/3b/2015. All experiments were performed in accordance with the relevant guidelines and regulations. 
Immunohistochemistry. For immunostaining, $30 \mu \mathrm{m}$ thick coronal hippocampal sections from all groups were processed simultaneously. Following permeabilization, sections were preincubated with $0.125 \mathrm{M} \mathrm{PB}$ containing 5\% (vol/vol) donkey serum and $0.3 \%$ Triton X-100 (TX-100) for $1 \mathrm{~h}$. Sections were incubated with primary antibodies diluted in $0.3 \% \mathrm{TX}-100$ at $4{ }^{\circ} \mathrm{C}$ for $48 \mathrm{~h}$, then washed and incubated in secondary antibodies for $2 \mathrm{~h}$, RT. A detailed description of the immunohistochemical procedure is listed in the Supplemental Information.

Electron microscopy. Following transcardial perfusion with a mixture of $2 \%$ formaldehyde (FA) and $2 \%$ glutaraldehyde in $0.1 \mathrm{M}$ phosphate buffer ( $\mathrm{PB}, \mathrm{pH}$ 7.4) under anesthesia, brains were post-fixed overnight in $4 \% \mathrm{FA}$ at $4{ }^{\circ} \mathrm{C}$. Ultrathin sections were examined with a JEM-1011 transmission electron microscope (JEOL, Tokyo, Japan). Data collection was performed blindly. A more detail description is found in the Supplemental Information.

Ultrastructural analysis. We analyzed six-seven mice per group. Visible bouton area, synaptic vesicles, active zones, mitochondria and their visible area were manually annotated in ImageJ $(1.48 \mathrm{v})$. A more detail description is found in the Supplemental Information.

Electrophysiology. For recordings of MF-fEPSPs and CA3-CA1 fEPSP, mice were anesthetized with isoflurane overdose and transcardially perfused with ice cold dissection artificial cerebrospinal fluid (ACSF) ${ }^{50}$ or directly decapitated, respectively. $350 \mu \mathrm{m}$-thick (MF recordings) or $300 \mu \mathrm{m}$-thick (CA3-CA1 recordings) hippocampal sections were incubated at $35^{\circ} \mathrm{C}$ for 30 minutes immediately after preparation and kept in a resting chamber at $22-24^{\circ} \mathrm{C}$ (MF recordings) or at RT (CA3-CA1 recordings) for at least an hour before use. A detailed description of all the electrophysiological experiments is found in the Supplemental Information.

Statistic. Statistic was performed with IBM SPSS Statistics version 25 (IBM) or with GraphPad Prism (GraphPad). Data distribution was assessed by Kolmogorov-Smirnov normality test and by inspecting histograms and normality Q-Q plots. Data were analyzed with one-way ANOVA followed by Tukey post-hoc test or by Kruskal-Wallis test followed by Mann Whitney U test, Bonferroni corrected with $\alpha$ set to 0.016667 to account for multiple comparisons, unless otherwise stated. Only two tail p-values were considered. Values are expressed as mean $\pm S E M, n$ indicates the number of boutons analyzed for EM analysis or the number of brain slices for electrophysiological experiments, unless otherwise stated. A detailed description of the statistical analysis is found in the Supplemental Information.

\section{Data availability}

The data that supports the findings of this study are available from the corresponding authors (VH and SJS) upon reasonable request.

Received: 10 September 2019; Accepted: 6 December 2019;

Published online: 23 December 2019

\section{References}

1. Bishop, N. A., Lu, T. \& Yankner, B. A. Neural mechanisms of ageing and cognitive decline. Nature 464, 529-535, https://doi. org/10.1038/nature08983 (2010).

2. Morrison, J. H. \& Hof, P. R. Life and death of neurons in the aging brain. Science 278, 412-419 (1997).

3. Burke, S. N. \& Barnes, C. A. Neural plasticity in the ageing brain. Nature reviews. Neuroscience 7, 30-40, https://doi.org/10.1038/ nrn1809 (2006).

4. Mattson, M. P. \& Magnus, T. Ageing and neuronal vulnerability. Nature reviews. Neuroscience 7, 278-294, https://doi.org/10.1038/ nrn1886 (2006)

5. Morrison, J. H. \& Baxter, M. G. The ageing cortical synapse: hallmarks and implications for cognitive decline. Nature reviews. Neuroscience 13, 240-250, https://doi.org/10.1038/nrn3200 (2012).

6. Rubinsztein, D. C., Marino, G. \& Kroemer, G. Autophagy and aging. Cell 146, 682-695, https://doi.org/10.1016/j.cell.2011.07.030 (2011).

7. Eisenberg, T. et al. Cardioprotection and lifespan extension by the natural polyamine spermidine. Nat Med 22, 1428-1438, https:// doi.org/10.1038/nm.4222(2016).

8. Glatigny, M. et al. Autophagy Is Required for Memory Formation and Reverses Age-Related Memory Decline. Curr Biol 29, 435-448 e438, https://doi.org/10.1016/j.cub.2018.12.021 (2019).

9. Gupta, V. K. et al. Restoring polyamines protects from age-induced memory impairment in an autophagy-dependent manner. Nature neuroscience 16, 1453-1460, https://doi.org/10.1038/nn.3512 (2013).

10. Pucciarelli, S. et al. Spermidine and spermine are enriched in whole blood of nona/centenarians. Rejuvenation Res 15, 590-595, https://doi.org/10.1089/rej.2012.1349 (2012).

11. Gupta, V. K. et al. Spermidine Suppresses Age-Associated Memory Impairment by Preventing Adverse Increase of Presynaptic Active Zone Size and Release. PLoS Biol 14, e1002563, https://doi.org/10.1371/journal.pbio.1002563 (2016).

12. Vivo, M. et al. Polyamines in the basal ganglia of human brain. Influence of aging and degenerative movement disorders. Neurosci Lett 304, 107-111, https://doi.org/10.1016/s0304-3940(01)01776-1 (2001).

13. Madeo, F., Eisenberg, T., Pietrocola, F. \& Kroemer, G. Spermidine in health and disease. Science 359, https://doi.org/10.1126/science. aan2788 (2018).

14. Madeo, F., Carmona-Gutierrez, D., Hofer, S. J. \& Kroemer, G. Caloric Restriction Mimetics against Age-Associated Disease: Targets, Mechanisms, and Therapeutic Potential. Cell Metab 29, 592-610, https://doi.org/10.1016/j.cmet.2019.01.018 (2019).

15. Wirth, M. et al. The effect of spermidine on memory performance in older adults at risk for dementia: A randomized controlled trial. Cortex 109, 181-188, https://doi.org/10.1016/j.cortex.2018.09.014 (2018).

16. Burke, S. N. \& Barnes, C. A. Senescent synapses and hippocampal circuit dynamics. Trends in neurosciences 33, 153-161, https://doi. org/10.1016/j.tins.2009.12.003 (2010).

17. Bartsch, T. \& Wulff, P. The hippocampus in aging and disease: From plasticity to vulnerability. Neuroscience 309, 1-16, https://doi. org/10.1016/j.neuroscience.2015.07.084 (2015). 
18. Lipinski, M. M. et al. Genome-wide analysis reveals mechanisms modulating autophagy in normal brain aging and in Alzheimer's disease. Proceedings of the National Academy of Sciences of the United States of America 107, 14164-14169, https://doi.org/10.1073/ pnas.1009485107 (2010).

19. Nicoll, R. A. \& Schmitz, D. Synaptic plasticity at hippocampal mossy fibre synapses. Nature reviews. Neuroscience 6, 863-876, https:// doi.org/10.1038/nrn1786 (2005).

20. Eisenberg, T. et al. Induction of autophagy by spermidine promotes longevity. Nat Cell Biol 11, 1305-1314, https://doi.org/10.1038/ ncb1975 (2009).

21. Stavoe, A. K., Gopal, P. P., Gubas, A., Tooze, S. A. \& Holzbaur, E. L. Expression of WIPI2B counteracts age-related decline in autophagosome biogenesis in neurons. Elife 8, https://doi.org/10.7554/eLife.44219 (2019).

22. Ojo, B. et al. Age-Induced Loss of Mossy Fibre Synapses on CA3 Thorns in the CA3 Stratum Lucidum. Neurosci J 2013, 839535, https://doi.org/10.1155/2013/839535 (2013).

23. Wilson, P. D. \& Franks, L. M. The effect of age on mitochondrial ultrastructure and enzymes. Adv Exp Med Biol 53, 171-183, https:// doi.org/10.1007/978-1-4757-0731-1_13(1975).

24. Rollenhagen, A. et al. Structural determinants of transmission at large hippocampal mossy fiber Synapses. Journal of Neuroscience 27, 10434-10444, https://doi.org/10.1523/Jneurosci.1946-07.2007 (2007).

25. Salin, P. A., Scanziani, M., Malenka, R. C. \& Nicoll, R. A. Distinct short-term plasticity at two excitatory synapses in the hippocampus. Proceedings of the National Academy of Sciences of the United States of America 93, 13304-13309 (1996).

26. Evstratova, A. \& Toth, K. Information processing and synaptic plasticity at hippocampal mossy fiber terminals. Front Cell Neurosci 8, 28, https://doi.org/10.3389/fncel.2014.00028 (2014).

27. Harris, K. M. \& Weinberg, R. J. Ultrastructure of synapses in the mammalian brain. Cold Spring Harb Perspect Biol 4, https://doi. org/10.1101/cshperspect.a005587 (2012).

28. Nicoll, R. A. \& Malenka, R. C. Contrasting properties of two forms of long-term potentiation in the hippocampus. Nature 377, 115-118, https://doi.org/10.1038/377115a0 (1995).

29. Barnes, C. A. Long-term potentiation and the ageing brain. Philos Trans R Soc Lond B Biol Sci 358, 765-772, https://doi.org/10.1098/ rstb.2002.1244 (2003).

30. Trovo, L. et al. Low hippocampal PI(4,5)P(2) contributes to reduced cognition in old mice as a result of loss of MARCKS. Nature neuroscience 16, 449-455, https://doi.org/10.1038/nn.3342 (2013).

31. Villanueva-Castillo, C., Tecuatl, C., Herrera-Lopez, G. \& Galvan, E. J. Aging-related impairments of hippocampal mossy fibers synapses on CA3 pyramidal cells. Neurobiol Aging 49, 119-137, https://doi.org/10.1016/j.neurobiolaging.2016.09.010 (2017).

32. Hylin, M. J. et al. A role for autophagy in long-term spatial memory formation in male rodents. J Neurosci Res $\mathbf{9 6}, 416-426$, https:// doi.org/10.1002/jnr.24121 (2018).

33. Pietrocola, F. et al. Spermidine induces autophagy by inhibiting the acetyltransferase EP300. Cell Death Differ 22, 509-516, https:// doi.org/10.1038/cdd.2014.215 (2015).

34. Morselli, E. et al. Spermidine and resveratrol induce autophagy by distinct pathways converging on the acetylproteome. J Cell Biol 192, 615-629, https://doi.org/10.1083/jcb.201008167 (2011).

35. Garcia-Prat, L. et al. Autophagy maintains stemness by preventing senescence. Nature 529, 37-42, https://doi.org/10.1038/ nature16187 (2016).

36. Applegate, M. D. \& Landfield, P. W. Synaptic vesicle redistribution during hippocampal frequency potentiation and depression in young and aged rats. The Journal of neuroscience: the official journal of the Society for Neuroscience 8, 1096-1111 (1988).

37. Weisskopf, M. G., Castillo, P. E., Zalutsky, R. A. \& Nicoll, R. A. Mediation of hippocampal mossy fiber long-term potentiation by cyclic AMP. Science 265, 1878-1882, https://doi.org/10.1126/science.7916482 (1994).

38. Castillo, P. E. et al. Rab3A is essential for mossy fibre long-term potentiation in the hippocampus. Nature 388, 590-593, https://doi. org/10.1038/41574 (1997).

39. Davis, R. L., Cherry, J., Dauwalder, B., Han, P. L. \& Skoulakis, E. The cyclic AMP system and Drosophila learning. Mol Cell Biochem 149-150, 271-278 (1995).

40. Maus, L. et al. Correlating Synaptic Ultrastructure and Function at the Nanoscale. 588848, https://doi.org/10.1101/588848\%JbioRxiv (2019).

41. Lawrence, J. J., Grinspan, Z. M. \& McBain, C. J. Quantal transmission at mossy fibre targets in the CA3 region of the rat hippocampus. J Physiol 554, 175-193, https://doi.org/10.1113/jphysiol.2003.049551 (2004).

42. Helassa, N. et al. Ultrafast glutamate sensors resolve high-frequency release at Schaffer collateral synapses. Proceedings of the National Academy of Sciences of the United States of America 115, 5594-5599, https://doi.org/10.1073/pnas.1720648115 (2018).

43. Oertner, T. G., Sabatini, B. L., Nimchinsky, E. A. \& Svoboda, K. Facilitation at single synapses probed with optical quantal analysis. Nature neuroscience 5, 657-664, https://doi.org/10.1038/nn867 (2002).

44. Deak, F. et al. Rabphilin regulates SNARE-dependent re-priming of synaptic vesicles for fusion. EMBO J 25, 2856-2866, https://doi. org/10.1038/sj.emboj.7601165 (2006).

45. Lonart, G. \& Sudhof, T. C. Region-specific phosphorylation of rabphilin in mossy fiber nerve terminals of the hippocampus. The Journal of neuroscience: the official journal of the Society for Neuroscience 18, 634-640 (1998).

46. Billups, B. \& Forsythe, I. D. Presynaptic mitochondrial calcium sequestration influences transmission at mammalian central synapses. The Journal of neuroscience: the official journal of the Society for Neuroscience 22, 5840-5847, 20026597 (2002).

47. David, G. \& Barrett, E. F. Mitochondrial Ca2 + uptake prevents desynchronization of quantal release and minimizes depletion during repetitive stimulation of mouse motor nerve terminals. J Physiol 548, 425-438, https://doi.org/10.1113/jphysiol.2002.035196 (2003).

48. Vos, M., Lauwers, E. \& Verstreken, P. Synaptic mitochondria in synaptic transmission and organization of vesicle pools in health and disease. Front Synaptic Neurosci 2, 139, https://doi.org/10.3389/fnsyn.2010.00139 (2010).

49. Jensen, J. R., Lynch, G. \& Baudry, M. Polyamines stimulate mitochondrial calcium transport in rat brain. J Neurochem 48, 765-772, https://doi.org/10.1111/j.1471-4159.1987.tb05583.x (1987).

50. Bischofberger, J., Engel, D., Li, L., Geiger, J. R. \& Jonas, P. Patch-clamp recording from mossy fiber terminals in hippocampal slices. Nat Protoc 1, 2075-2081, https://doi.org/10.1038/nprot.2006.312 (2006).

\section{Acknowledgements}

The authors are grateful for the support by staff members of the animal facilities of the Leibniz Forschungsinstitut für Molekulare Pharmakologie (FMP), the Charité Universitätsmedizin and the Institute of Molecular Biosciences. We thank Silke Zillmann and Sabine Hahn, Tünde Magyar and Renáta Pop for technical assistance. We thank Dr. Constance Scharff for the use of the Agilent Technologies Stratagene Mx3005P Real-time PCR system and the NeuroCure imaging facility for the use of the Leica SP8 confocal microscope. The Project is supported by the Bundesministerium für Bildung und Forschung to SJS (Smartage, 01GQ1420A), to EP (Smartage 01GQ1420D), to DS and NM (Smartage 01GQ1420B) and to VH (Smartage 01GQ1420C), by the Deutsche Forschungsgemeinschaft (Collaborative Research Grant SFB958, A03) to SJS, by the European Union and co-financed by the European Social Fund (grant agreement no. EFOP-3.6.1-16-2016-00024). BR was also 
supported by the UNKP Bolyai+ fellowship of the Hungarian Ministry of Human Capacities and by the János Bolyai Research Fellowship of the Hungarian Academy of Sciences. FM is grateful to the Austrian Science Fund FWF (SFB LIPOTOX F3007 \& F3012, W1226, P29203, P29262, P27893, P31727), the Austrian Federal Ministry of Education, Science and Research and the University of Graz for grants "Unkonventionelle Forschung-InterFast and Fast4Health" as well as "flysleep" (BMWFW-80.109/0001-WF/V/3b/2015) and the field of excellence program BioHealth. We acknowledge support from NAWI Graz and the BioTechMed-Graz flagship project "EPIAge".

\section{Author contributions}

M.M., G.K., A.S., A.W., F.M., T.M., N.M., E.P., D.S., V.H. and S.J.S. designed research. M.M., G.K., T.E., A.S., A.W., A.Z., F.E.M., R.B., L.M.V., R.P.S. and D.T. performed research. M.M., G.K., A.S., A.W., E.M., A.Z., F.E.M., L.M.V., R.P.S., S.J.H. and D.T. analyzed data. T.E. and F.M. contributed to reagents. M.M., V.H. and S.J.S. wrote the paper.

\section{Competing interests}

F.M., S.J.S. and T.E. have equity interests in T.L.L. (The Longevity Labs), a company founded in 2016 that will develop natural food extracts. All other authors declare no competing interests.

\section{Additional information}

Supplementary information is available for this paper at https://doi.org/10.1038/s41598-019-56133-3.

Correspondence and requests for materials should be addressed to V.H. or S.J.S.

Reprints and permissions information is available at www.nature.com/reprints.

Publisher's note Springer Nature remains neutral with regard to jurisdictional claims in published maps and institutional affiliations.

(c) (i) Open Access This article is licensed under a Creative Commons Attribution 4.0 International License, which permits use, sharing, adaptation, distribution and reproduction in any medium or format, as long as you give appropriate credit to the original author(s) and the source, provide a link to the Creative Commons license, and indicate if changes were made. The images or other third party material in this article are included in the article's Creative Commons license, unless indicated otherwise in a credit line to the material. If material is not included in the article's Creative Commons license and your intended use is not permitted by statutory regulation or exceeds the permitted use, you will need to obtain permission directly from the copyright holder. To view a copy of this license, visit http://creativecommons.org/licenses/by/4.0/.

(C) The Author(s) 2019 